\title{
The CORALIE survey for southern extra-solar planets
}

\section{The very low-mass companions of HD 141937, HD 162020, HD 168443 and HD 202206: Brown dwarfs or "superplanets"?^}

\author{
S. Udry, M. Mayor, D. Naef, F. Pepe, D. Queloz, N. C. Santos, and M. Burnet \\ Observatoire de Genève, $51 \mathrm{ch}$. des Maillettes, 1290 Sauverny, Switzerland \\ Received 26 February 2002 / Accepted 22 April 2002

\begin{abstract}
Doppler CORALIE measurements of the solar-type stars HD 141937, HD 162020, HD 168443 and HD 202206 show Keplerian radial-velocity variations revealing the presence of 4 new companions with minimum masses close to the planet/brown-dwarf transition, namely with $m_{2} \sin i=9.7,14.4,16.9$, and $17.5 M_{\text {Jup }}$, respectively. The orbits present fairly large eccentricities $(0.22 \leq e \leq 0.43)$. Except for HD 162020, the parent stars are metal rich compared to the Sun, as are most of the detected extra-solar planet hosts. Considerations of tidal dissipation in the short-period HD 162020 system points towards a brown-dwarf nature for the low-mass companion. HD 168443 is a multiple system with two low-mass companions being either brown dwarfs or formed simultaneously in the protoplanetary disks as superplanets. For HD 202206, the radial velocities show an additional drift revealing a further outer companion, the nature of which is still unknown. Finally, the stellar-host and orbital properties of massive planets are examined in comparison to lighter exoplanets. Observed trends include the need of metal-rich stars to form massive exoplanets and the lack of short periods for massive planets. If confirmed with improved statistics, these features may provide constraints for the migration scenario.
\end{abstract}

Key words. techniques: radial velocities - stars: binaries: spectroscopic - stars: individual: HD 141937 - stars: individual: HD 162020 - stars: individual: HD 168443 - stars: individual: HD 202206

\section{Introduction}

Since the discovery of the extra-solar planet orbiting $51 \mathrm{Peg}$ (Mayor \& Queloz 1995), high-precision radial-velocity measurements proved to be very efficient for detecting very lowmass companions to solar-type stars. In about 6 years, close to 80 planetary candidates with minimum mass $m_{2} \sin i<10 M_{\text {Jup }}$ have been announced including 7 planetary systems and a few sub-Saturnian planets (see e.g. Udry \& Mayor 2001; Fischer et al. 2002, for recent reference updates).

Interestingly, brown-dwarf candidates, easier to detect with high-precision Doppler surveys, seem to be more sparse than exoplanets (Mayor et al. 1997), especially in the 10-30 $M_{\text {Jup }}$ interval (Halbwachs et al. 2000b), the so-called brown-dwarf desert. Objects in this domain are very important to understand the brown-dwarf/planet transition. The paradigm behind the distinction between planets and brown dwarfs may rely on different considerations: mass, physics of the interior, formation mechanism, etc. From the "formation" point of view, the brown-dwarf companions belong to the low-mass end of

Send offprint requests to: S. Udry,

e-mail: stephane.udry@obs.unige.ch

* Based on observations collected with the CORALIE echelle spectrograph on the 1.2-m Euler Swiss telescope at La Silla Observatory, ESO Chile. the secondaries formed in binary stars whereas planets form in the protostellar disk. Such distinct origins of planetary and multiple-star systems is clearly emphasized by the two peaks in the observed distribution of minimum masses of secondaries to solar-type stars as shown in Fig. 1 (top), providing an updated ${ }^{1}$ version of the diagram. They strongly suggest different formation and evolution histories for the two populations. Below $10 M_{\text {Jup }}$ the planetary distribution increases with decreasing mass and is thus not the tail of the stellar binary distribution.

For the objects detected by the radial-velocity technique, only minimum masses are determined because the inclination angles of the orbital planes relative to the line of sight cannot be derived from the spectroscopic data only. The determination of the true masses for most of these objects is expected soon with high-angular resolution astrometric facilities that will become available in the upcoming years (e.g. PRIMA on the VLTI, SIM). It is however already possible to apply a statistical deconvolution to the growing sample of exoplanet candidates (Jorissen et al. 2001; Zucker \& Mazeh 2001). The updated planetary true mass distribution, derived as in

1 All candidates known on the 15th of November 2001. The masses are from the discovery papers or from CORALIEELODIE orbital solutions. A summary table is provided at obswww . unige.ch/ naef/who_discovered_that_planet.html 

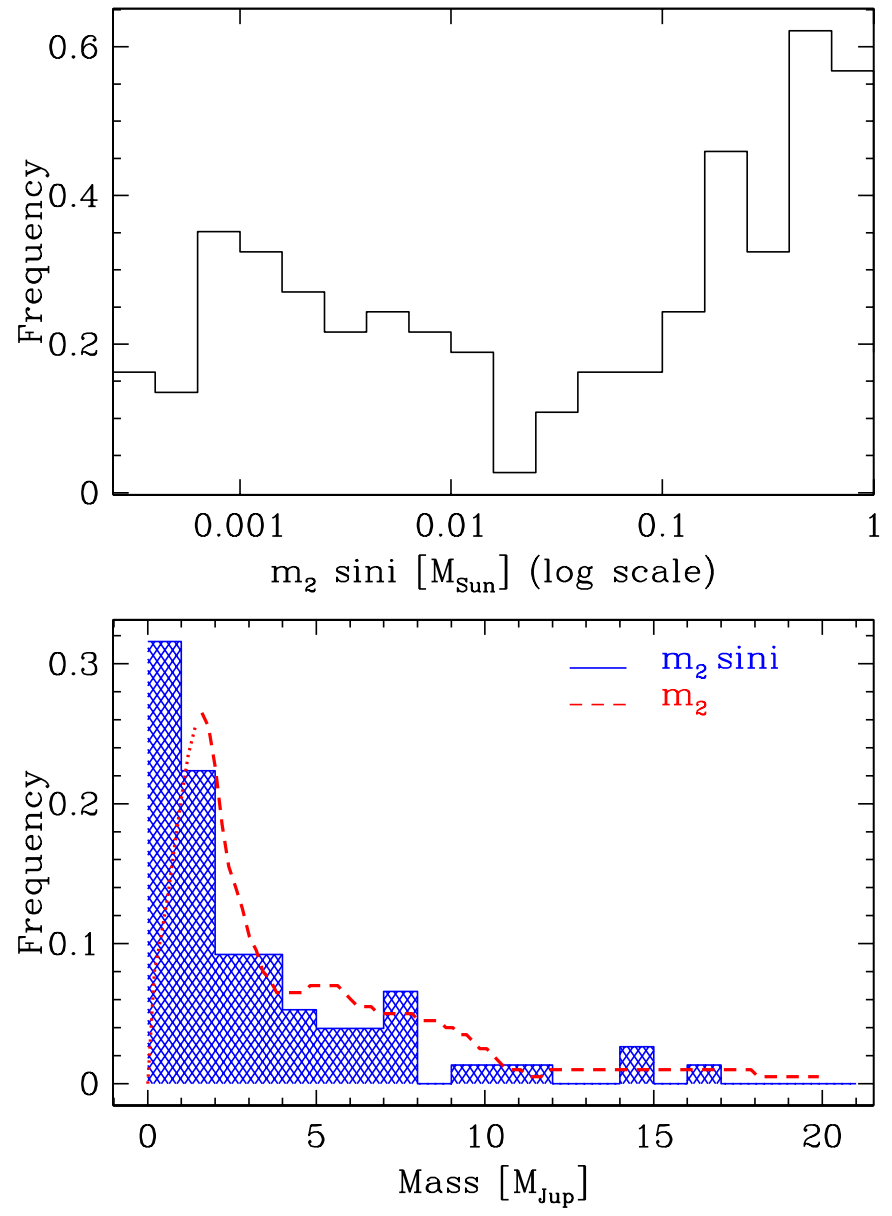

Fig. 1. Top: observed distribution of minimum masses of secondaries to solar-type stars (log scale). Stellar binaries with $\mathrm{G}$ and $\mathrm{K}$ primaries are from Duquennoy \& Mayor (1991) and Halbwachs et al. (2000a). Bottom: updated statistical distribution of true giant-planet masses (dashed line; derived as in Jorissen et al. 2001) superimposed on the $m_{2} \sin i$ planetary distribution.

Jorissen et al. (2001), is presented in Fig. 1 (bottom). Probably because of the strong observational bias favouring the more massive planets, the distribution looks "bimodal". A careful treatment of the bias, however, has to be done before being able to convincingly interpret the shape of the distribution. Nevertheless, the bias at the high-mass end of the planetary distribution is vanishing and the observational maximum mass of exoplanets is fairly well determined, around $10-11 M_{\text {Jup }}$ where the curve drops to almost zero. If this value is correct then the question of the true masses and nature of the candidates with slightly higher values of $m_{2} \sin i$ is becoming very interesting.

Since the summer of 1998, a large high-precision radialvelocity programme has been carried out with the CORALIE echelle spectrograph on the 1.2-m Euler Swiss telescope at La Silla (Queloz et al. 2000b; Udry et al. 2000a). The CORALIE survey has been very successful, with the detection of a significant fraction of the known exoplanet candidates (Udry \& Mayor 2001). Information on the method, technical and instrumental details are given in Baranne et al. (1996). Recent improvements in the reduction software have allowed us to bring the long-term instrumental precision of individual measurements from the previously obtained $\sim 7 \mathrm{~m} \mathrm{~s}^{-1}$ down to $\sim 2 \mathrm{~m} \mathrm{~s}^{-1}$ (Queloz et al. 2001b). Asteroseismology measurements of $\alpha$ Cen A even show a short-term precision below the $1 \mathrm{~m} \mathrm{~s}^{-1}$ limit over 1 night (Bouchy \& Carrier 2001). The size of the telescope is now the main limitation of the precision actually achieved for most of our sample stars.

The cross-correlation technique used has proven to be very simple, robust and efficient for radial-velocity measurements. However, it is still not optimum in terms of Doppler information extraction from the spectra (Bouchy et al. 2001; Chelli 2000). Recently, Pepe et al. (2002) improved the procedure by introducing a "correct" weighting of the spectral lines involved in the cross correlation. At the same time they also reduced the astroclimatic-induced noise by restricting more severely the zone of the spectra potentially affected by telluric lines. The overall gain corresponds to a virtual increase in the signal-tonoise by a factor of $\sim 1.25$ (i.e. a virtual decrease of the photonnoise error by the same factor).

This new procedure for radial-velocity estimation is used for the objects described in this paper. We present 4 new very low-mass companions to solar-type stars, detected with CORALIE, and with minimum masses in the planet-brown $d$ warf transition domain. With four other objects - HD 114762 (Latham et al. 1989), HD $110833^{2}$ (Mayor et al. 1997), HD 39091 (Hugh et al. 2002) and HD 136118 (Fischer et al. 2002) - these candidates are the only companions to solar-type stars known to date with minimum masses between $\sim 10$ and $20 M_{\text {Jup }}$. They are thus of prime importance for the description of the transition zone between brown dwarfs and planets. The first sections of the paper are dedicated to the description of the stellar properties of the hosts of the new candidates, then to their orbital characteristics and to a discussion of the possible nature of these objects. Finally, the orbital properties and the characteristics of stars with "massive" planets are examined in comparison with systems harbouring "lighter" planets.

The radial-velocity data for the new candidates will be made available in electronic form at the CDS via anonymous ftp to cdsarx.u-strasbg. fr (130.17.128.5) or via http://cdsweb.u-strasbg.fr/cgi-bin/qcat?]/A+A/390/267.

\section{Stellar characteristics of the candidate hosts}

The stars hosting the 4 very low-mass candidates presented here were observed by the HIPPARCOS astrometric satellite. Most of the quoted photometric and astrometric parameters are thus taken from the mission output catalogue (ESA 1997). High-precision spectroscopic studies of these stars have also been performed by several authors in the context of examining the metallicity distribution of stars hosting planets in comparison to "single" stars of the solar neighbourhood (Gonzalez et al. 2001; Santos et al. 2001a,b). Observed and inferred stellar parameters from these different sources are summarized in Table 1. In the table, the given masses of primary stars are estimated from evolutionary tracks of the Geneva models with appropriate spectroscopic parameters $\left(T_{\mathrm{eff}}, L\right.$, metallicity;

\footnotetext{
${ }^{2}$ HD 110833 was shown to be a stellar binary by Halbwachs et al. (2000b) using the HIPPARCOS astrometric data.
} 
Table 1. Observed and inferred stellar parameters for HD 141937, HD 162020, HD 168443 and HD 202206. Photometric and astrometric parameters are from HIPPARCOS (ESA 1997). The atmospheric parameters $T_{\text {eff }}, \log g,[\mathrm{Fe} / \mathrm{H}]$ are from Santos et al. (2001a,b) and Gonzalez et al. (2001). Spectral types are from HIPPARCOS or derived from spectroscopy. The bolometric correction is computed from Flower (1996) ${ }^{a}$ using the spectroscopic $T_{\text {eff }}$ determinations. The projected rotational velocities come from a calibration of the CORALIE cross-correlation functions. Activity indicators $\left(S_{\mathrm{COR}}, \log R_{\mathrm{HK}}^{\prime}\right)$ are estimated from $N_{\text {act }}$ high $S / N$ CORALIE spectra, following Santos et al. (2000). The given ages are derived from the $\log R_{\mathrm{HK}}^{\prime}$ activity indicator (Donahue 1993) or/and from the Geneva evolutionary models (Schaller et al. 1992; Schaerer et al. 1993) which also provide mass estimates. Finally, rotational periods are obtained from the $\log R_{\mathrm{HK}}^{\prime}$ as well, following Noyes et al. (1984).

\begin{tabular}{|c|c|c|c|c|c|}
\hline Parameter & & HD 141937 & HD 162020 & HD 168443 & HD 202206 \\
\hline Spectral Type & & G2/G3V & $\mathrm{K} 3 \mathrm{~V}$ & $\mathrm{G} 8 \mathrm{IV}^{b}$ & G6V \\
\hline$V$ & & 7.25 & 9.10 & 6.92 & 8.08 \\
\hline$B-V$ & & 0.628 & 0.964 & 0.724 & 0.714 \\
\hline$\pi$ & [mas] & $29.89 \pm 1.08$ & $31.99 \pm 1.48$ & $26.40 \pm 0.85$ & $21.58 \pm 1.14$ \\
\hline$M_{V}$ & & 4.63 & 6.63 & 4.03 & 4.75 \\
\hline $\mathrm{BC}$ & & -0.055 & -0.388 & -0.125 & -0.082 \\
\hline$L$ & {$\left[L_{\odot}\right]$} & 1.17 & 0.25 & 2.17 & 1.07 \\
\hline$[\mathrm{Fe} / \mathrm{H}]$ & & 0.11 & $0.01 \pm 0.11$ & $0.10 \pm 0.03$ & $0.37 \pm 0.07$ \\
\hline$M$ & {$\left[M_{\odot}\right]$} & 1.1 & 0.75 & $1.01^{b}$ & 1.15 \\
\hline$T_{\text {eff }}$ & {$[\mathrm{K}]$} & 5925 & $4830 \pm 80$ & $5555 \pm 40$ & $5765 \pm 40$ \\
\hline $\log g$ & {$[\mathrm{cgs}]$} & 4.62 & $4.76 \pm 0.25$ & $4.10 \pm 0.12$ & $4.75 \pm 0.20$ \\
\hline$v \sin i$ & {$\left[\mathrm{~km} \mathrm{~s}^{-1}\right]$} & 2.1 & 1.9 & 1.7 & 2.5 \\
\hline$N_{\text {act }}$ & & 19 & - & 1 & - \\
\hline$S_{\mathrm{COR}}$ & & $0.23 \pm 0.04$ & - & 0.19 & - \\
\hline$S_{\mathrm{MW}}$ & & 0.24 & - & 0.21 & - \\
\hline $\log \left(R_{\mathrm{HK}}^{\prime}\right)$ & & -4.65 & - & $-4.8\left(-5.08^{b}\right)$ & - \\
\hline$P_{\text {rot }}\left(R_{\mathrm{HK}}^{\prime}\right)$ & [days] & 13.25 & - & $26.8\left(37^{b}\right)$ & - \\
\hline age $\left(R_{\mathrm{HK}}^{\prime} /\right.$ Model $)$ & [Gyr] & $1.6 / 2 \pm 6$ & $-/ 6 \pm 18$ & $7.8^{b} />10$ & $-/ 5.6 \pm 1.2$ \\
\hline
\end{tabular}

${ }^{a}$ Quoted values in the paper include errors. The correct values have been obtained directly from the author.

${ }^{b}$ Value quoted in Marcy et al. (1999).

Schaller et al. 1992; Schaerer et al. 1993). Age estimates are also provided by those models but usually with very large uncertainties for our type of stars. The projected rotational velocity, $v \sin i$, comes from the calibration of the CORALIE crosscorrelation functions ${ }^{3}(\mathrm{CCF})$ derived in the same way as the calibration of the ELODIE CCF (Queloz et al. 1998).

\subsection{HD 141937 (HIP 77740)}

From the HIPPARCOS parallax $(29.89 \pm 1.08$ mas $)$ and visual magnitude $(V=7.25)$, we derive for HD 141937 an absolute magnitude $M_{V}=4.63$ in agreement with its given G2V spectral type and color index $(B-V=0.628)$. Santos et al. (2001b) performed a high-resolution spectroscopic abundance study for this star and derived precise values for its effective temperature $\left(T_{\mathrm{eff}}=5925 \mathrm{~K}\right)$, metallicity $([\mathrm{Fe} / \mathrm{H}]=0.11)$ and gravity $(\log g=4.62)$, using a standard local thermodynamical equilibrium (LTE) analysis. Using a calibrated bolometric correction $B C=-0.055$ (Flower 1996) combined with the spectroscopic $T_{\text {eff }}$ determination, the star luminosity is found to be $L=1.17 L_{\odot}$. A mass $M=1.1 M_{\odot}$ and a badly constrained age of $2 \pm 6 \mathrm{Gyr}$ are then estimated from the Geneva evolutionary models (Schaller et al. 1992).

3 The calibration does not account for metallicity effects. For metalrich stars the rotational broadening is therefore slightly overestimated.
The dispersion of the HIPPARCOS photometric data $\left(\sigma_{\mathrm{Hp}}=0.007 \mathrm{mag}\right)$ shows no evidence of variation of the star luminosity at the instrument precision. The same conclusion holds from the Geneva photometry observations.

\section{2. $H D 162020$ (HIP 87330)}

In the HIPPARCOS catalogue HD 162020 is a K2 dwarf with $V=6.35$ and $B-V=0.964$. The catalogue also lists a precise astrometric parallax $\pi=31.99 \pm 1.48$ mas corresponding to a distance of $31.26 \mathrm{pc}$ from the Sun. The derived absolute magnitude $M_{V}=6.63$ is typical for a K3 dwarf. From high-resolution CORALIE spectra, Santos et al. (2001a) derived the following spectroscopic parameters: $T_{\mathrm{eff}}=4830 \mathrm{~K},[\mathrm{Fe} / \mathrm{H}]=0.01$ and $\log g=4.76$ (Table 1). With a calibrated bolometric correction $B C=-0.388$ (Flower 1996) and the derived effective temperature the star luminosity is found to be $L=0.25 L_{\odot}$, also suggesting a K3V spectral type. Solar-metallicity models (Schaller et al. 1992) give then a mass of $0.75 M_{\odot}$. They also point towards an old stellar age but with a very large uncertainty, much larger than the age itself $(6 \pm 18 \mathrm{Gyr})$. On the other hand, we will see further that the activity level of the star rather suggests a younger age.

The dispersion of the HIPPARCOS photometric data of $\mathrm{HD} 162020\left(\sigma_{\mathrm{Hp}}=0.018 \mathrm{mag}\right)$ is found to be slightly higher 
than the value expected for a 9th-magnitude star. We will see below that the star shows spectral indications of activity that can explain this feature.

\subsection{HD 168443 (HIP 89844, GJ 4052)}

The stellar characteristics of HD 168443 have been discussed by Marcy et al. (1999) in the paper announcing the detection of the first planet orbiting the star. From the HIPPARCOS photometric data, they have estimated the star to reside $\sim 1.5 \mathrm{mag}$ above the main sequence in the HR diagram $\left(M_{V}=4.03\right)$, being slightly evolved. They estimate the star to have a G8IV spectral type and a mass of $1.01 M_{\odot}$. A recent high-precision spectroscopic study by Gonzalez et al. (2001) completely corroborates that result by deriving reliable values of the effective temperature $\left(T_{\text {eff }}=5555 \mathrm{~K}\right)$, metallicity $([\mathrm{Fe} / \mathrm{H}]=0.1)$ and gravity $(\log g=4.1)$ of the star. Using Flower (1996) calibration for the bolometric correction $(B C=-0.125)$, one obtains a luminosity $L=2.17 L_{\odot}$. The mass derived from the Geneva evolutionary tracks (Schaller et al. 1992) agrees with the estimate of Marcy et al. (1999) and the age is found to be larger than $10 \mathrm{Gyr}$, suitable for a slightly evolved late $\mathrm{G}$ star. These main stellar properties are recalled in Table 1.

The star is found to be photometrically stable in both the HIPPARCOS data $\left(\sigma_{\mathrm{Hp}}=0.007 \mathrm{mag}\right)$ and the Geneva photometry $\left(\sigma_{V}=0.005 \mathrm{mag}\right)$.

\subsection{HD 202206 (HIP 104903)}

In the HIPPARCOS catalogue, HD 202206 is given as a G6 dwarf of visual magnitude $V=8.08$ and color index $B-V=$ 0.714 . The measured parallax $(21.58 \pm 1.14$ mas $)$ leads to an absolute magnitude $M_{V}=4.75, \sim 0.4$ mag brighter than the expected value for a typical G6 dwarf of solar metallicity. From CORALIE spectra, Santos et al. (2001a) derived an effective temperature $T_{\text {eff }}=5765 \mathrm{~K}$, a gravity $\log g=4.75$ and a very high metal content $[\mathrm{Fe} / \mathrm{H}]=0.37$ (Table 1 ). The very high metallicity of HD 202206 probably accounts for its overluminosity as $T_{\text {eff }}$ is also larger than the value expected for a G6 dwarf. From $B C=-0.082$, we derive $L=1.07 L_{\odot}$ and the Geneva models (Schaerer et al. 1993) yields a mass of $M=1.15 M_{\odot}$ and an age of about $5.6 \pm 1.2 \mathrm{Gyr}$.

As for HD 162020, the dispersion of the HIPPARCOS photometric data of HD $202206\left(\sigma_{\mathrm{Hp}}=0.013 \mathrm{mag}\right)$ is a bit high for the star magnitude but again some indication of stellar activity is seen in the spectra (see below).

\subsection{Chromospheric activity}

The amplitude of the radial-velocity jitter associated with intrinsic stellar activity may reach a few tens of $\mathrm{m} \mathrm{s}^{-1}$, especially for high-rotation stars with large spectral-line asymmetry due to spots (Saar \& Donahue 1997). The coherent-spot survival on the stellar surface over several rotational periods may even mimic the radial-velocity variation induced by a planetary companion (Queloz et al. 2001a).
The stellar activity can be associated with the presence of chromospheric emission in the centre of the $\mathrm{Ca}$ II $\mathrm{H}$ and $\mathrm{K}$ absorption lines. When reported to the photospheric flux, the intensity of this emission provides good quantitative estimators $\left(S_{\mathrm{COR}}{ }^{4}\right.$ or $\left.\log R_{\mathrm{HK}}^{\prime}\right)$ of the activity level of solar-type stars and thus to the expected level of induced spurious noise on the radial-velocity measurements (Saar et al. 1998; Santos et al. 2000). For the brightest stars in our planet-search sample, such indicators are directly measured on the CORALIE spectra. However, the low brightness of some of the targets does not allow us to derive good indicators ${ }^{5}$ (as e.g. for HD 162020 and HD 202206; Santos et al. 2000). In such cases, the available spectra are added to obtain a high signal in the $\lambda$ 3968.5 $\AA$ Ca II $\mathrm{H}$ absorption line region. The resulting spectrum may contain traces of the thorium-argon spectrum used as a radial-velocity reference (Baranne et al. 1996) and is thus not optimal for precise spectroscopic studies in this region but it at least allows for a visual check of the chromospheric emission in the center of the line. Figure 2 shows the corresponding spectral domain for the stars presented in this paper.

Moderate values of the $\log R_{\mathrm{HK}}^{\prime}$ chromospheric activity indicator have been derived for HD $141937(-4.65)$ and HD $168443\left(-4.8^{6}\right)$ (Table 1; see Santos et al. 2000 for details about the technique). Moreover, the corresponding $\lambda 3968.5 \AA \mathrm{Ca}$ II H absorption lines do not show clear chromospheric emission features (Fig. 2). Radial-velocity jitters are thus not expected to be large for these 2 stars although the effect might be slightly increased for the early $G$ dwarf HD 141937 showing a non-zero projected rotational velocity $\left(v \sin i=2.1 \mathrm{~km} \mathrm{~s}^{-1}\right)$.

Although too faint to provide a reliable estimate of $\log R_{\mathrm{HK}}^{\prime}$, HD 162020 clearly shows a strong emission feature in the core of the $\mathrm{Ca}$ II $\mathrm{H}$ line. The activity-related radial-velocity jitter of a slowly rotating $\mathrm{K} 2 \mathrm{dwarf}$ is however not expected to be large (Santos et al. 2000). It is certainly not responsible for the large radial-velocity variation observed for HD $162020\left(3.3 \mathrm{~km} \mathrm{~s}^{-1}\right.$ peak to peak). As shown by Henry (1999), activity could on the other hand be invoked to explain the dispersion of the HIPPARCOS photometric data. The activity level points towards a young age for the star. However, we will see in Sect. 4.1.1 that the system has probably been synchronized over the lifetime of the star, thus increasing the stellar rotation. As activity is very sensitive to rotation, the activity level could also have been boosted by the synchronization making the star look younger than its real age.

Although noticeable, the activity level of HD 202206 is not very important (Fig. 2) and should not cause any trouble

\footnotetext{
${ }^{4}$ The $S_{\mathrm{COR}}$ activity index has been calibrated to the Mount-Wilson system index $S_{\mathrm{MW}}$ (Vaughan et al. 1978) to compute the $\log R_{\mathrm{HK}}^{\prime}$ indicator (Noyes et al. 1984).

5 The correction for the diffuse light in the spectrograph is not known precisely enough and for low $S / N$ spectra it represents a significant fraction of the light in the line. It then induces systematic errors in the estimate of the activity indicators.

${ }^{6}$ Our estimate only rests on 1 high- $S / N$ spectrum. Marcy et al. (1999) quote a more reliable value of -5.08 suggesting that HD 168443 is an even more quiet star.
} 


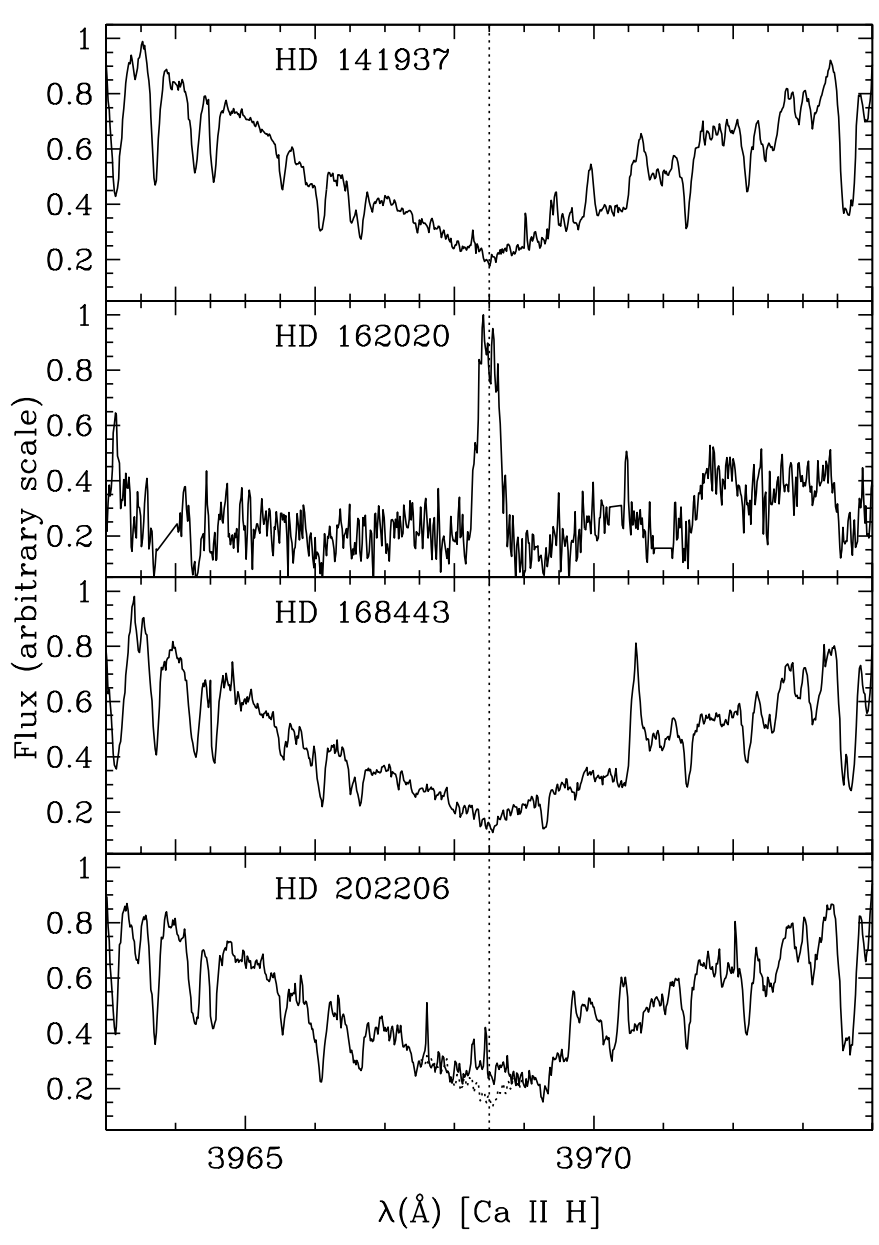

Fig. 2. $\lambda 3968.5 \AA \mathrm{Ca}$ II $\mathrm{H}$ absorption line region of the summed CORALIE spectra for the 4 stars considered in this paper. Clear emission features are observed for HD 162020 and HD 202206. For the latter a small part of the HD 168443 spectrum in the center of the line has also been reported in the diagram (dotted line) to emphasize its chromospheric emission. For clarity, spurious emission contamination features of the thorium-argon lamp have been removed from the spectrum of HD 162020. No trace of chromospheric emission is visually observed for HD 141937 and HD 168443, in agreement with their moderate measured values of $\log R_{\mathrm{HK}}^{\prime}$ (Table 1 ).

beyond adding some low-level high-frequency spurious noise in the radial-velocity measurements.

Finally, from the $\log R_{\mathrm{HK}}^{\prime}$ value (when available), following the calibration by Noyes et al. (1984) we can estimate the rotational period of the star as well as the stellar age using the calibration in Donahue (1993) (also quoted in Henry et al. 1996). The inferred statistical values are given in Table 1 . The so-derived ages are compatible with values provided by evolutionary tracks, taking into account the large uncertainties in the age determinations.

\section{HD 141937 orbital solution}

Over 882 days, we obtained 81 CORALIE observations of HD 141937, with a photon-noise uncertainty distribution peaking around $6 \mathrm{~m} \mathrm{~s}^{-1}$. A fairly large long-term radial-velocity variation was noticed early on, but we had to wait for more than one orbital period to derive good orbital parameters because the star was unfortunately behind the Sun at the time of the maximum and minimum of the radial-velocity curve. The best Keplerian fit to the data yields a precise period $P=653.2$ days, an eccentricity $e=0.41$ and a semi-amplitude $K=234.5 \mathrm{~m} \mathrm{~s}^{-1}$ (Table 2). The radial-velocity curve is displayed in Fig. 3 with the residuals around the solution. The mass $M=1.1 M_{\odot}$ derived for the parent star leads to a planet minimum mass $m_{2} \sin i=9.7 M_{\text {Jup }}$.

The weighted rms around the best Keplerian solution $\left(\sigma(O-C)=8.7 \mathrm{~m} \mathrm{~s}^{-1}\right)$ is large compared to the typical measurement uncertainties $\left(\chi_{\text {red }}^{2}=2.69\right)$. As no longer-term variation clearly arises from the residuals of the fit (Fig. 3), the reason for the slight extra noise $\left(\sim 6 \mathrm{~m} \mathrm{~s}^{-1}\right)$ probably lies in the star activity $\left(\log R_{\mathrm{HK}}^{\prime}=-4.65\right)$ coupled with the observed nonzero stellar rotation $\left(v \sin i=2.1 \mathrm{~km} \mathrm{~s}^{-1}\right)$, in agreement with values quoted by Saar et al. (1998) or Santos et al. (2000).

\section{HD 162020 b: A "Hot Brown Dwarf"}

Between the 24th of June 1999 and the 14th of October 2001, 46 CORALIE radial velocities of HD 162020 were gathered. The low brightness of the star limits the photon noise of our measurements to about $8 \mathrm{~m} \mathrm{~s}^{-1}$ in a typical integration time of 15 minutes, under normal weather conditions. As the observed radial-velocity variation was large, several observations were made under worse meteorological conditions and the distribution of measurement errors shows a right-end tail up to $40 \mathrm{~m} \mathrm{~s}^{-1}$. The short orbital period and large observed semiamplitude of the radial-velocity variation allowed us, however, to very rapidly ${ }^{7}$ determine orbital elements and characteristics of the companion. The imprecise subset of measurements limit the quality of the solution $\left(\mathrm{rms} \simeq 13.6 \mathrm{~m} \mathrm{~s}^{-1}, \chi_{\text {red }}^{2}=2.46\right)$, and we have derived a new solution with only the 30 CORALIE observations with photon-noise errors below $10 \mathrm{~m} \mathrm{~s}^{-1}$. This solution yields a short period $P$ of $8.428198 \pm 0.000056$ days with a non-zero eccentricity $e=0.277 \pm 0.002$. Taking $0.75 M_{\odot}$ as the primary mass (see above), the derived orbital parameters lead to a minimum mass $m_{2} \sin i=14.4 M_{\text {Jup }}$ for the companion. The very short orbital period derived for HD 162020 locates the companion only $0.074 \mathrm{AU}$ from the primary star. At such a small distance from its parent star and following e.g. Guillot et al. (1996), the companion equilibrium temperature at the surface is estimated to be around $650 \mathrm{~K}$. The companion is thus a "hot" superplanet or brown dwarf on a non-circular orbit. The complete set of orbital elements with their uncertainties is given in Table 2 as well as some interesting inferred quantities.

The weighted rms to the Keplerian fit is $8.1 \mathrm{~m} \mathrm{~s}^{-1}$ and the reduced $\chi^{2}$ of the solution is 1.37 . The improvement from the preliminary solution including all measurements clearly shows the significant degrading effect of the lower-quality velocities on the derived solution. The residuals around the solution show no significant evidence of a possible long-period additional companion (Fig. 4). Although the star shows clear indication of chromospheric activity, the level of activity-induced extra noise

\footnotetext{
7 The discovery was announced on the 4th of May 2000 by an ESO press release (ESO 2000).
} 


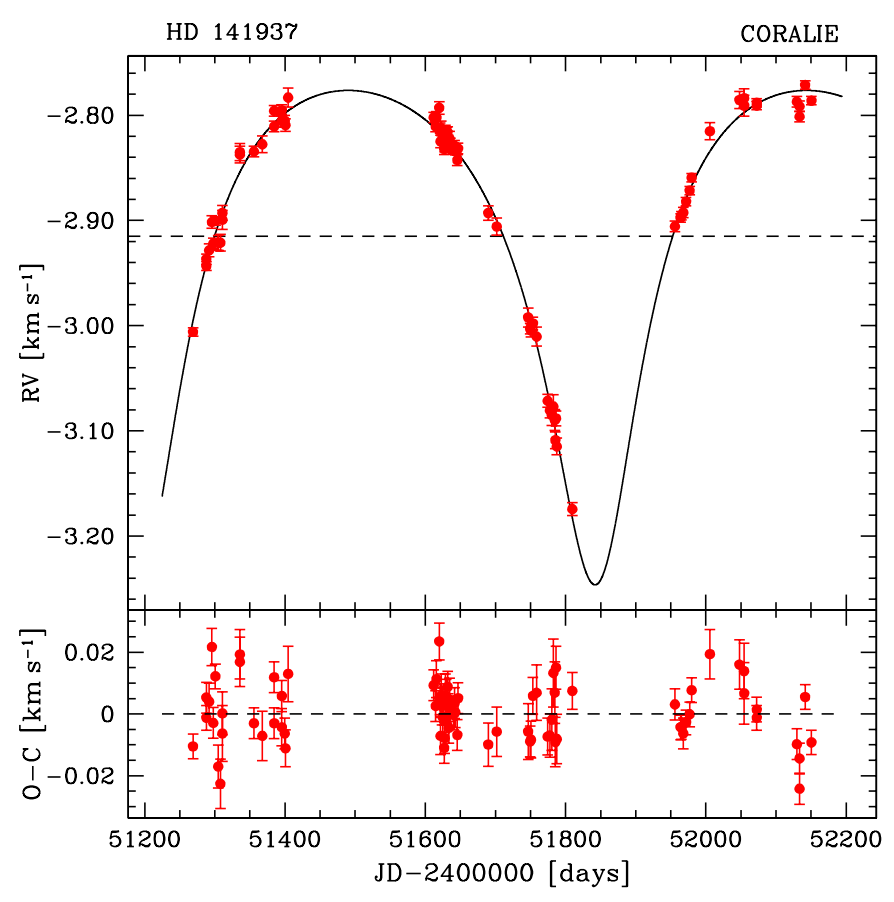

Fig. 3. Top. CORALIE radial-velocity measurements of HD 141937 superimposed on the best Keplerian model. Error bars (photon noise) are very small in comparison with the amplitude of the radial-velocity variation. Bottom. Residuals around the solution.

is very small, in agreement with values predicted for slowly rotating K dwarfs (Saar et al. 1998; Santos et al. 2000).

\subsection{Tidal dissipation in the HD 162020 system}

In the same way as close binaries, giant gaseous planets closely orbiting their stars are subject to spin-orbit synchronization and/or orbital circularization associated with tidal dissipation in the star or the planet. The tilted mass distribution induced in the convective envelope of the object by the gravitational attraction of the companion is phase shifted because of dissipation. It then exerts a torque on the companion, leading to an exchange of angular momentum between its spin and the orbital motion, tending to synchronize and circularize the orbit. When the tidally distorted star has a convective envelope, the tidal dissipation may be represented by the viscosity of convective eddies (Zahn 1989).

For binaries with dwarf-star primaries, orbits with periods smaller than $\sim 10$ days are circularized (see e.g. Mayor et al. 2001, for a recent review). Much attention has also been paid to this effect for hot Jupiters (Rasio et al. 1996; Marcy et al. 1997; Ford et al. 1999). We will focus here on the case of HD 162020 in which the "planet" is slightly more distant than the one orbiting 51 Peg but also much heavier.

\subsubsection{Tidal dissipation in the star and synchronization}

Following the treatment of Zahn $(1989,1992)$ for objects with a convective envelope, in the case of not too high eccentricities,

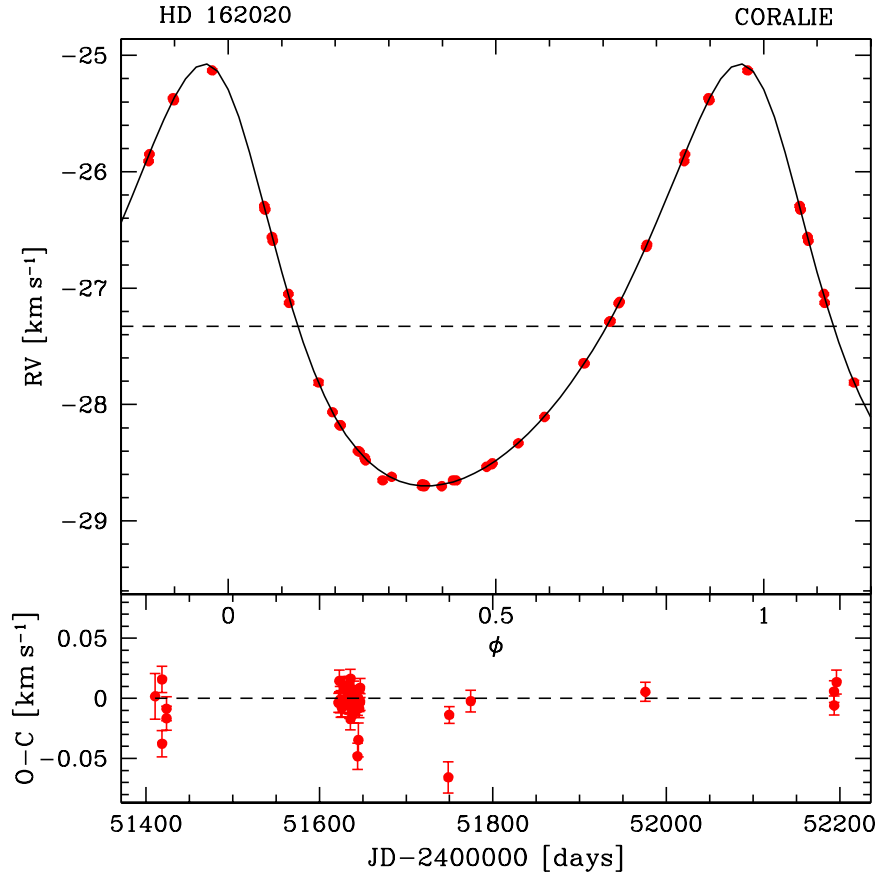

Fig. 4. Top. Phased CORALIE radial-velocity measurements and Keplerian orbital solution for HD 162020. The solution is calculated with only the 30 higher signal-to-noise measurements but all the 46 radial velocities with photon-noise errors (error bars) are reported on the diagram. Bottom. Residuals around the solution displayed as a function of time.

the time scale for synchronization by tidal dissipation in the star through convective viscosity is given by

$$
\frac{1}{\tau_{\mathrm{sync}}}=6 \frac{\lambda_{2}}{t_{f}} q^{2} \frac{M_{\star} R_{\star}^{2}}{I_{\star}}\left(\frac{R_{\star}}{a}\right)^{6}
$$

where $q=m_{\mathrm{pl}} / M_{\star}$ is the planet to star mass ratio, $R_{\star}$ and $I_{\star}$ are the stellar radius and moment of inertia, $a$ is the orbital separation, $t_{f}$ is the characteristic time of the physical process responsible for the dissipation (turnover timescale of the eddies) and $\lambda_{2}$ is close to the apsidal constant measuring the response to the external torque imposed by the companion when the star is fully convective and smaller when the star has a finite convective zone. Zahn (1994) has tabulated $k^{2}=I_{\star} /\left(M_{\star} R_{\star}^{2}\right), t_{f}$ and $\lambda_{2}$ for the zero-age main sequence. Interpolating in his table, with $M_{\star}=0.75 M_{\odot}$, we obtain for HD 162020: $k^{2}=0.132$, $t_{f}=0.509 \mathrm{yr}$ and $\lambda_{2}=0.0094$. The typical time $t_{f}$ is usually defined as $t_{f}=\left(M_{\star} R_{\star}^{2} / L_{\star}\right)^{1 / 3}$ (Zahn 1994). In the case of nonfully convective dwarfs, an estimate of

$t_{f}^{\prime}=\left(M_{\mathrm{env}} R_{\mathrm{env}}\left(R_{\star}-R_{\mathrm{env}}\right) / 3 L_{\star}\right)^{1 / 3}$,

where $M_{\text {env }}$ is the mass of the convective envelope and $R_{\text {env }}$ the radius at its base, is more appropriate (Rasio et al. 1996). For $\operatorname{HD} 162020\left(M_{\mathrm{env}} / M_{\star} \simeq 0.17\right.$, Murray et al. $2001, R_{\mathrm{env}} / R_{\star} \simeq$ 0.7 ), we calculate $t_{f}^{\prime} \simeq 0.13 \mathrm{yr}$. With $q=0.01834 / \sin i$, we derive

$\tau_{\text {sync }}($ HD 162020 $) \simeq 1.3 \times 10^{10} \sin ^{2} i$ yr.

Even taking into account our limited knowledge of the viscous dissipative process and the large uncertainty of the stellar 
age determination, it appears that HD 162020 can be synchronized by the close-orbiting low-mass companion. In such a case, when the two components are close, the synchronized state may be unstable to orbital decay (Hut 1980). Instability occurs when the ratio of spin to orbital angular momentum $J_{\text {spin }} / J_{\text {orb }}>1 / 3$. For HD $162020, J_{\text {spin }} / J_{\text {orb }} \simeq 0.007$. Hence, the star was brought into synchronicity without reaching tidal instability.

In the case of synchronization, knowing the stellar rotational period and assuming that the orbital and rotation axes coincide $^{8}$, the equatorial velocity $V_{\text {eq }}$ may be derived from the star radius and then the orbital plane inclination is obtained from the measured projected rotational velocity. Using a typical value $R_{\star} \simeq 0.75 R_{\odot}$ for a K 3 dwarf, we get $V_{\text {eq }} \simeq 4.5 \mathrm{~km} \mathrm{~s}^{-1}$ and an indicative $\sin i \simeq 0.42\left(v \sin i=1.9 \mathrm{~km} \mathrm{~s}^{-1}\right)$. This leads to $m_{2} \simeq 34 M_{\text {Jup }}$ i.e. a probable brown dwarf for the companion of HD 162020 . Of course, the uncertainty on the $v \sin i$ value is difficult to estimate and probably it does not exclude the companion from having a low stellar mass.

\subsubsection{Circularization due to tidal dissipation in the star}

The observed significant orbital eccentricity of HD 162020 ( $e=$ $0.277 \pm 0.002$ ) shows that the circularization induced by tidal dissipation in the stellar convective envelope had no time to proceed over the age of the star. Assuming again standard tidal dissipation theory (e.g. Zahn 1989; Verbunt \& Phinney 1995), an explicit expression for the circularization time is given by

$\frac{1}{\tau_{\mathrm{cir}}} \equiv-\frac{\mathrm{d} \ln e}{\mathrm{~d} t}=\frac{f}{t_{f}^{\prime}} \frac{M_{\mathrm{env}}}{M_{\star}} \frac{1}{q(1+q)}\left(\frac{R_{\star}}{a}\right)^{8}$

leading to

$\tau_{\text {cir }}(\mathrm{HD} 162020) \simeq 4.5 \times 10^{9} \frac{\sin i}{f} \mathrm{yr}$.

The parameters are the same as above and $f$ is obtained by integrating viscous dissipation of tidal energy throughout the convective zone. $f \simeq 1$ as long as $t_{f}^{\prime} \ll P_{\text {orb }}$ (e.g. Zahn \& Bouchet 1989; Verbunt \& Phinney 1995; Rasio et al. 1996; Ford et al. 1999) corresponding to the case where the main contribution to the viscosity comes from the largest convective cells. As is clearly explained in Rasio et al. (1996), for $P_{\text {orb }}<t_{f}^{\prime}$, the largest eddies can no longer contribute to the viscosity because the velocity field they are damping will have changed direction before they can transfer momentum. Only eddies with turnover times smaller than the pumping period $\left(P_{\text {orb }} / 2\right)$ will contribute and the eddy viscosity is then reduced by a factor $\left(2 t_{f}^{\prime} / P_{\mathrm{orb}}\right)^{\alpha}$. The value of $\alpha$ is debated but generally thought to be 1 (Zahn 1992) or 2 (Goldreich \& Keeley 1977). So, in general we have

$f=f^{\prime} \min \left[1,\left(\frac{P_{\text {orb }}}{2 t_{f}^{\prime}}\right)^{\alpha}\right]$

\footnotetext{
${ }^{8}$ As observed e.g. for HD 209458 (Queloz et al. 2000a). However, it should be pointed out that, in the case of a brown-dwarf companion, this may not be the case.
}

with $f^{\prime} \simeq 1$. Choosing $\alpha=2$ (the extreme case), we estimate then for $\operatorname{HD} 162020 f \simeq 0.015$ and the circularization time becomes

$\tau_{\text {cir }}(\mathrm{HD} 162020) \simeq 3 \times 10^{11} \sin i \mathrm{yr}$.

Even taking into account the above rough estimate of $\sin i$, the tidal dissipation in the convective envelope of HD 162020 is thus not supposed to have circularized the orbit. On the other hand, the derived $\tau_{\text {cir }}$ value does not allow for a very small value of the $\sin i$.

\subsubsection{Tidal dissipation in the planet}

Can tides in the low-mass companion have been more efficient than stellar tides in circularizing the orbit, as it is the case for 51 Peg (Rasio et al. 1996)? In this case, the typical circularization time is given by

$\tau_{\mathrm{e}}=\frac{4}{63} Q\left(\frac{a^{3}}{G M_{\star}}\right)^{1 / 2} q\left(\frac{a}{R_{\mathrm{pl}}}\right)^{5}$

(Rasio et al. 1996, and quoted references), where $Q$ is proportional to the tidal pumping period $\left(P_{\text {orb }} / 2\right)$ and is about $10^{5}$ for Jupiter. Comparing 51 Peg and HD 162020 we can write

$$
\begin{aligned}
\frac{\tau_{\mathrm{e}}^{51 \text { Peg }}}{\tau_{\mathrm{e}}^{162020}} & =\frac{P_{\mathrm{orb}}^{51 \mathrm{Peg}}}{P_{\mathrm{orb}}^{162020}}\left(\frac{a^{51 \text { Peg }}}{a^{162020}}\right)^{13 / 2}\left(\frac{M_{\star}^{162020}}{M_{\star}^{51 \text { Peg }}}\right)^{3 / 2} \\
& \times \frac{m_{\mathrm{pl}}^{51 \mathrm{Peg}}}{m_{\mathrm{pl}}^{162020}}\left(\frac{R_{\mathrm{pl}}^{162020}}{R_{\mathrm{pl}}^{51 \mathrm{Peg}}}\right)^{5} \mathrm{yr} .
\end{aligned}
$$

From the value quoted in Rasio et al. (1996) we obtain

$$
\tau_{\mathrm{e}}(\mathrm{HD} 162020) \simeq 2 \times 10^{12} \frac{\sin i(162020)}{\sin i(51 \mathrm{Peg})} \mathrm{yr} .
$$

The tides in the low-mass companion were thus not able to circularize the orbit over the lifetime of the star which is expected from the observed non-zero value of the orbital eccentricity.

In conclusion, it appears that the companion of HD 162020 is probably a brown dwarf (although a low-mass star cannot be ruled out). The observed eccentricity is so not surprising if the system was formed as for usual binary stars. This system will be a perfect target for future high-angular resolution astrometric facilities. It potentially will provide the true mass of a low-mass brown dwarf.

\subsection{Photometric transit search}

The short orbital period makes HD 162020 a good target for a photometric transit search. The star was intensively followed with the Danish SAT at La Silla in collaboration with our colleagues from Copenhagen, unfortunately without success. This is not a surprise in view of the considerations developed in the previous sections about the probable orbital inclination of the system. The photometric monitoring will be presented in a forthcoming paper (Olsen et al. in prep) with other candidates followed. 
Table 2. CORALIE best Keplerian orbital solutions derived for HD 141937, HD 162020 and HD 202206 as well as inferred planetary parameters. Note that the parameter uncertainties are directly taken from the diagonal elements of the covariance matrix as if the parameters were uncorrelated. These uncertainties are thus probably underestimated

\begin{tabular}{llccc}
\hline \hline \multicolumn{2}{l}{ Parameter } & HD 141937 & HD 162020 & HD 202206 \\
\hline$P$ & [days] & $653.22 \pm 1.21$ & $8.428198 \pm 0.000056$ & $256.003 \pm 0.062$ \\
$T$ & {$[\mathrm{JD}-2400000]$} & $51847.38 \pm 1.97$ & $51990.677 \pm 0.005$ & $51919.02 \pm 0.16$ \\
$e$ & & $0.41 \pm 0.01$ & $0.277 \pm 0.002$ & $0.429 \pm 0.002$ \\
$V$ & {$\left[\mathrm{~km} \mathrm{~s}^{-1}\right]$} & $-2.915 \pm 0.002$ & $-27.328 \pm 0.002$ & $14.681 \pm 0.002$ \\
$\omega$ & {$[\mathrm{deg}]$} & $187.72 \pm 0.80$ & $28.40 \pm 0.23$ & $160.32 \pm 0.31$ \\
$K$ & {$\left[\mathrm{~m} \mathrm{~s}^{-1}\right]$} & $234.5 \pm 6.4$ & $1813 \pm 4$ & $564.8 \pm 1.3$ \\
Linear drift & {$\left[\mathrm{m} \mathrm{s}^{-1} \mathrm{yr}^{-1}\right]$} & - & - & $42.9 \pm 1.3$ \\
$N_{\text {meas }}$ & & 81 & 30 & 95 \\
$\sigma(\mathrm{O}-\mathrm{C})$ & {$\left[\mathrm{m} \mathrm{s}^{-1}\right]$} & 8.7 & 8.1 & 9.5 \\
$\chi_{\text {red }}^{2}$ & & 2.69 & 1.37 & 2.38 \\
\hline$a_{1} \sin i$ & {$[\mathrm{AU}]$} & 0.01282 & 0.00135 & 0.012 \\
$f(m)$ & {$\left[10^{-6} M_{\odot}\right]$} & 0.658 & 4.620 & 3.526 \\
$m_{2} \sin i$ & {$\left[M_{\text {Jup }}\right]$} & 9.7 & 14.4 & 17.5 \\
$a$ & {$[\mathrm{AU}]$} & 1.52 & 0.074 & 0.83 \\
$T_{\text {eq }}$ & {$[\mathrm{K}]$} & - & 650 & - \\
\hline
\end{tabular}

\section{HD 168443: Superplanets in disks?}

The inner planet orbiting HD 168443 was detected by Marcy et al. (1999). They had gathered 30 HIRES/Keck radial-velocity measurements over 800 days that allowed them to characterize a 58-d period orbit from which they inferred a $5 M_{\text {Jup }}$ companion to the star. These authors also mentioned a significant drift ( $89.4 \mathrm{~m} \mathrm{~s}^{-1} \mathrm{yr}^{-1}$ ) of the observed velocities indicating the presence of an additional outer companion in the system, as yet undetected directly. Their careful check for a possible stellar companion to HD 168443, in spectroscopic, adaptive optics and HIPPARCOS astrometric measurements, allowed them to constrain a potential stellar companion to be at a distance between 5 and $30 \mathrm{AU}$ from the primary star and with a mass smaller than $0.5 M_{\odot}$. Marcy et al. (1999) also mentioned a clear indication of curvature in the radial-velocity drift that allowed them to postulate that the $2^{\text {nd }}$ companions had to be on an orbit with a period of at least 4 years and be more massive than $15 M_{\mathrm{Jup}}$.

After the announcement of the detection of a planet orbiting HD 168443, the star - part of our planet-search programme in the southern hemisphere - has been followed regularly with CORALIE. We gathered 58 additional observations over 670 days reaching the 2nd extremum of the curve of radial-velocity variation due to the 2 nd companion. Combining our own measurements with the 30 published Keck velocities (Marcy et al. 1999) we obtained the complete description of the system for the IAU 202 symposium on Planetary Systems in the Universe where we presented the simultaneously-derived Keplerian solutions for the 2 companions (Udry et al. 2000b). The second companion was found to be on a 1667-day orbit and have a $m_{2} \sin i$ of $15.1 M_{\text {Jup }}$. This early solution is recalled in Table 3.

The star was of course closely followed by Marcy and collaborators as well. Within a few weeks they derived a complete solution with their own data (Marcy et al. 2001) confirming our early result. On our side we also went on gathering
14 additional CORALIE observations in 195 days. In Fig. 5, we present an updated two-Keplerian complete solution of the system derived from the 72 CORALIE velocities and the early 30 Marcy et al. (1999) measurements. The derived parameters are given in Table 3 . The quality of the fit is fair $\left(\chi_{\text {red }}^{2}=2.46\right)$. The increase of the monitoring span since the first proposed solution allowed us and Marcy et al. (2001) to better cover the longperiod orbit, estimated now to have a period of $P=1739$ days (1770 days for Marcy et al. 2001), slightly longer than the first estimate. Setting the primary mass to $1 M_{\odot}$, the minimum masses of the inner and outer companions inferred from our model are now 7.7 and $16.9 M_{\mathrm{Jup}}$, respectively.

\subsection{Nature of the companion to HD 168443 ?}

The properties of HD 168443, if not due to unlikely orbitalplane inclinations for both companions, set very interesting questions on the nature and possible formation of such systems. Are the companions of HD 168443 superplanets formed in the protoplanetary disk, brown dwarfs or even low-mass stars?

The hierarchical organization of the system does not allow stability criteria to set constraining upper limits for the companions masses. Marcy et al. (2001) have shown that even a triple stellar system can be stable in the case of a coplanar geometry. Different orientations of the two orbital planes favour, however, substellar companions. From the HIPPARCOS astrometric measurements Marcy et al. (2001) also estimate the maximum mass of the outer component to be smaller than $\sim 42 M_{\text {Jup }}$.

If formed outside the disk, the more massive outer companion is close enough to have truncated the disk within the ice limit, preventing thus a giant planet from forming in the outer regions and then moving towards the system center as predicted by the migration scenario. The inner companion has then also to be a brown dwarf. On the other hand, if the two 


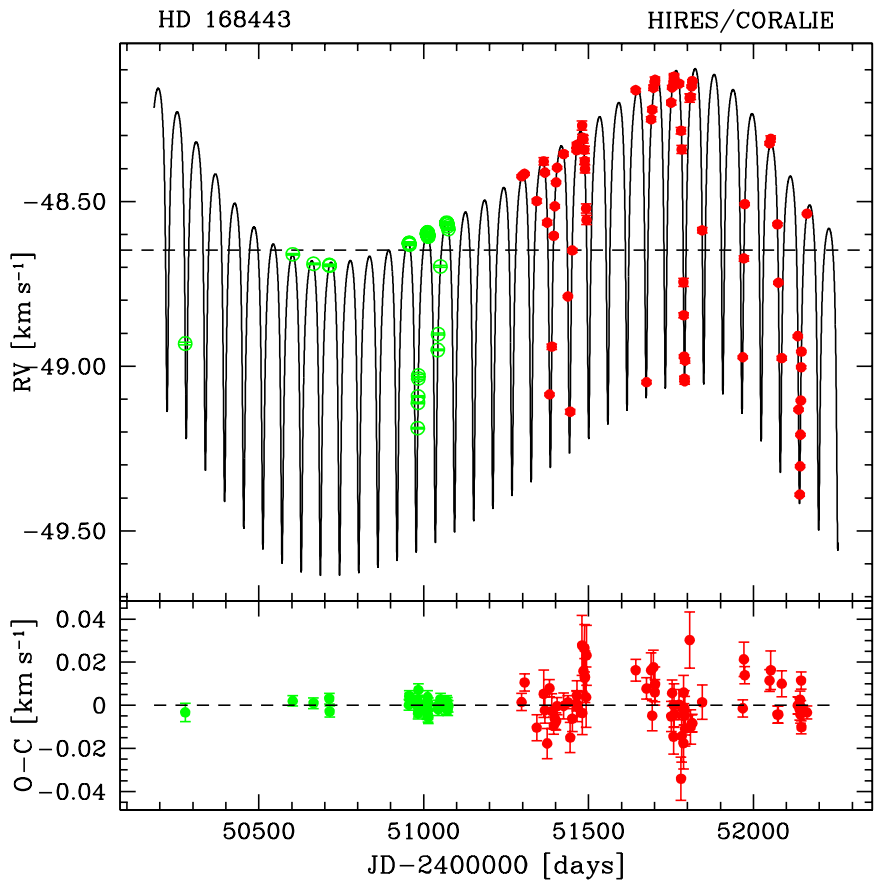

Fig. 5. Top. Simultaneous two-Keplerian solution for the HD 168443 system derived from older Keck velocities (from Marcy et al. 1999, open symbols) and subsequent CORALIE measurements (filled circles). Bottom. Residuals around the combined solution.

companions are in the disk, they are located well within the ice limit of the young protoplanetary disk. According to the migration scenario both objects had thus to move simultaneously towards the central region of the system. Simulations by Kley (2000) explore this possibility and show that in such a case, the outer object accreates disk material more efficiently. This rises the possibility of creating superplanets in the disk with masses larger than $15 M_{\text {Jup }}$. In such a case, if not very rare, these objects with masses above the high-mass tail of the observed planet-mass distribution (Fig. 1; Jorissen et al. 2001) would represent a new population in the diagram. This seems to be rarely observed. The "final" answer will be given by future precise astrometric measurements that will determine the true masses of the companions.

\section{HD 202206: Triple system or superplanet in a binary?}

The CORALIE observations of HD 202206 started in August 1999. The obvious variation of the radial velocities allowed us to announce the detection of a low-mass companion to the star already after one orbital period, in May 2000 (ESO 2000), at the same time as HD 162020 also described in this paper. When a second maximum of the radial-velocity curve was reached we noticed a slight drift of its value. We have now gathered 95 measurements covering more than 3 orbital periods. A simultaneous fit of a Keplerian model and a linear drift yields a period of 256 days and a large semi-amplitude of $565 \mathrm{~m} \mathrm{~s}^{-1}$ for the orbital solution (Table 2; Fig. 6). The orbit is fairly eccentric with $e=0.429$.

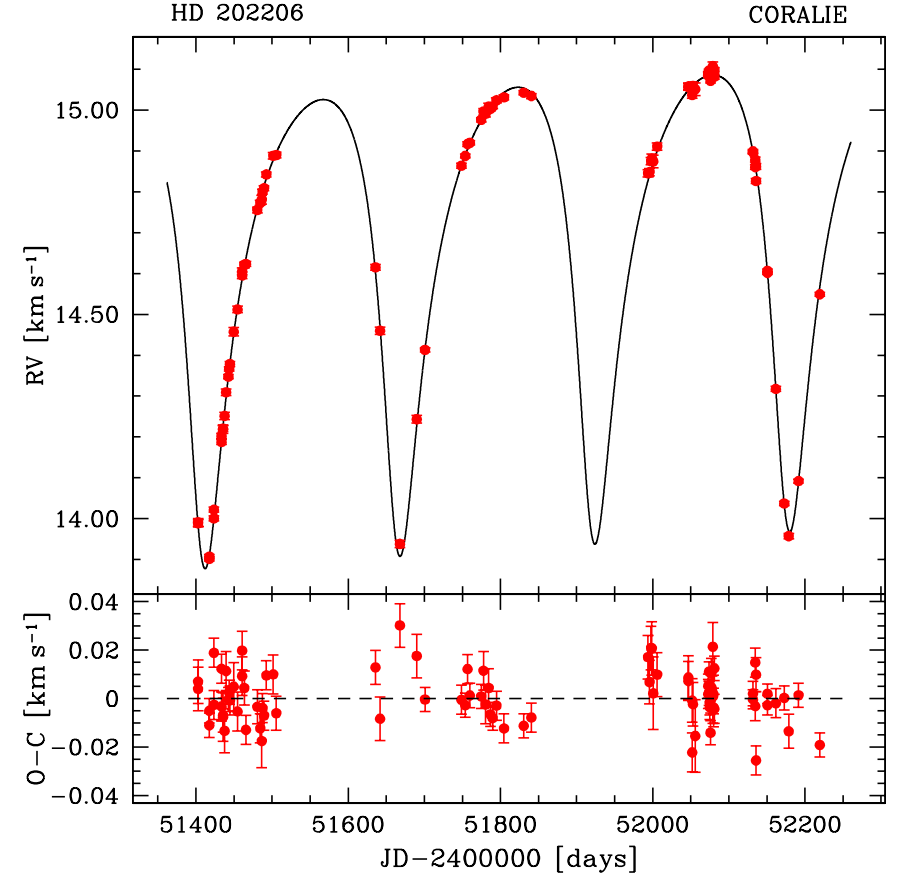

Fig. 6. Top. CORALIE temporal radial-velocity measurements of HD 202206 (except observations with photon-noise error larger than $15 \mathrm{~m} \mathrm{~s}^{-1}$ ), superimposed on the best model including a Keplerian orbital solution + a linear radial-velocity drift with $42.9 \mathrm{~m} \mathrm{~s}^{-1} \mathrm{yr}^{-1}$ slope. Bottom. Residuals around the combined solution.

Choosing the stellar mass to be $1.15 M_{\odot}$ (see above), the inferred minimum mass for the secondary is $17.5 \mathrm{M}_{\mathrm{Jup}}$.

The quality of the solution is good with a weighted rms of $9.5 \mathrm{~m} \mathrm{~s}^{-1}$ around the fitted model (to be compared to the typical photon-noise error of $8 \mathrm{~m} \mathrm{~s}^{-1}$ ) and a reduced $\chi^{2}$ value of 2.38 .

The slope of the radial-velocity drift is found to be $42.88 \mathrm{~m} \mathrm{~s}^{-1} \mathrm{yr}^{-1}$. The available two older CORAVEL measurements obtained in 1989 and 1991 unfortunately do not allow us to further constrain the longer-period companion.

Contrary to HD 110833 which was detected with a comparable $m_{2} \sin i$ companion (Mayor et al. 1997) and then shown to be in reality a stellar binary (Halbwachs et al. 2000b), the distance of HD 202206 (46.3 pc) prevents the HIPPARCOS astrometric data from constraining the visual orbit. At such a distance the expected displacement on the sky of the star due to the inner companion is only 0.26 mas, supposing $\sin i=1$. A factor of 5 on the $\sin i$ bringing the companion into the stellar domain would still be insufficient at the HIPPARCOS precision.

If not due to unfavourable orbital inclination, the observed low secondary mass sets the companion close to the limit of the planetary and brown-dwarf domains. The fairly large measured eccentricity does not help us to further constrain the nature of the object as most of the extrasolar "massive" planetary candidates are found on elongated orbits. An often proposed explanation for the planet eccentricities involves the gravitational perturbation of a stellar companion, that can be applied to HD 202206.

A more interesting characteristic of the system is given by the star's very high metallicity $([\mathrm{Fe} / \mathrm{H}]=0.37)$. Santos et al. (2001a) have shown that a large fraction of stars with this level 
Table 3. Same as Table 2 but for HD 168443. The early IAU 202 complete solution is given as well as the new updated one.

\begin{tabular}{|c|c|c|c|c|c|}
\hline \multirow{2}{*}{\multicolumn{2}{|c|}{ Parameter }} & HD $168443 \mathrm{~b}$ & HD $168443 \mathrm{c}$ & HD 168443 b & HD $168443 \mathrm{c}$ \\
\hline & & \multicolumn{2}{|c|}{ IAU 202 (Udry et al. 2000b) } & \multicolumn{2}{|c|}{ Updated orbit } \\
\hline$P$ & [days] & $58.117 \pm 0.014$ & $1667 \pm 48$ & $58.116 \pm 0.001$ & $1739.50 \pm 3.98$ \\
\hline$T$ & [JD-2400000] & $51558.36 \pm 0.12$ & $50269.5 \pm 36.0$ & $51616.36 \pm 0.02$ & $52014.5 \pm 3.6$ \\
\hline$e$ & & $0.526 \pm 0.008$ & $0.265 \pm 0.049$ & $0.529 \pm 0.002$ & $0.228 \pm 0.005$ \\
\hline$V$ & {$\left[\mathrm{~km} \mathrm{~s}^{-1}\right]$} & \multicolumn{2}{|c|}{$-48.744 \pm 0.002$} & \multicolumn{2}{|c|}{$-48.647 \pm 0.002$} \\
\hline$\omega$ & {$[\mathrm{deg}]$} & $172.2 \pm 1.1$ & $59 \pm 5$ & $171.61 \pm 0.22$ & $63.67 \pm 0.84$ \\
\hline$K$ & {$\left[\mathrm{~m} \mathrm{~s}^{-1}\right]$} & $473 \pm 6$ & $288 \pm 13$ & $475.7 \pm 1.3$ & $293.8 \pm 2.3$ \\
\hline$N_{\text {mes }}$ & & \multicolumn{2}{|c|}{$30^{a}+58^{b}$} & \multicolumn{2}{|c|}{$30^{a}+72^{b}$} \\
\hline$\sigma(\mathrm{O}-\mathrm{C})$ & {$\left[\mathrm{m} \mathrm{s}^{-1}\right]$} & \multicolumn{2}{|c|}{7.6} & \multicolumn{2}{|c|}{$3.0^{a} / 8.1^{b}$} \\
\hline$\chi_{\mathrm{red}}^{2}$ & & \multicolumn{2}{|c|}{-} & \multicolumn{2}{|c|}{2.46} \\
\hline$a_{1} \sin i$ & {$[\mathrm{AU}]$} & 0.002138 & 0.04559 & 0.002157 & 0.04574 \\
\hline$f(m)$ & {$\left[10^{-6} M_{\odot}\right]$} & 0.386 & 4.164 & 0.396 & 4.218 \\
\hline$m_{2} \sin i$ & {$\left[M_{\mathrm{Jup}}\right]$} & 7.2 & 15.1 & 7.7 & 16.9 \\
\hline$a$ & {$[\mathrm{AU}]$} & 0.29 & 2.67 & 0.29 & 2.85 \\
\hline
\end{tabular}

${ }^{a}$ HIRES data, $\quad{ }^{b}$ CORALIE measurements.

of metal content have giant-planet companions. Once again, precise astrometric measurements will clarify the problem.

\section{Discussion}

Several scenarios may be invoked for the formation of companions to solar-type stars with masses in the planet/brown-dwarf transition domain. On the one hand, the observations of very light free-floating objects in young formation regions (Zapatero Osorio et al. 2000; Lucas \& Roche 2000) suggest that the minimum mass of brown dwarfs formed as stars by fragmentation of a protostellar cloud could be very small, at the $\sim 5 M_{\text {Jup }}$ level. On the other hand, several processes have been proposed for the formation of massive planets in stellar accretion disks: by gravitational instability of the disk (possibly triggered by the perturbation of an additional stellar companion, Boss 2000, 2001) or by gas accretion of simultaneously migrating planets trapped into resonances (e.g. Kley 2000). The interesting point in the latter scenarios is that a supplementary companion - stellar or planetary - is required or at least is supposed to enhance the process.

\subsection{The "companion's" influence}

To check if the potential companion's influence can be seen in the data, we made an inventory of the known visual companions of the planet-hosting stars. This visual companion census was done through the Simbad-Vizir database (star within 1 arcmin) and through the recent literature on adaptive-optics programmes searching for faint companions to stars bearing planets (Lloyd et al. 2000; Luhman \& Jayawardhana 2002). We also looked for indication of additional radial-velocity drifts in the known planetary orbital solutions, mentioned in the discovery papers or appearing in our CORALIE data. The census is probably far from being exhaustive but nevertheless can bring initial insight into the question. The ratio of the number of stars hosting planets with visual or spectroscopic additional companions to the number of stars without further companions, in $2-M_{\text {Jup }}$ smoothing windows, is shown in the lower panel of Fig. $7^{9}$. Unlike what was expected from the abovementioned scenarios, there is no evidence that additional companions would favour massive-planet formation. There is even a trend for lower-mass planets $\left(m_{2} \sin i \leq 4 M_{\text {Jup }}\right.$, solid line $)$ to appear more often in "multiple" systems than massive ones ( $m_{2} \sin i>4 M_{\text {Jup }}$, dashed line), by a factor of roughly 2 . The statistics become too poor above $9 M_{\text {Jup }}$ to be able to draw conclusions about the more massive candidates.

Is this observed difference significant? The upper panel of Fig. 7 presents the cumulative functions of the planetary mass distributions for stars hosting planets a. without further visual or spectroscopic companion (solid line), $b$. with a visual companion (dotted line), $c$. with an additional radial-velocity drift (long-dash line) unveiling a second planetary or stellar companion and $d$. with a visual or a spectroscopic companion (i.e. $b$ or $c$, short-dash line). From these curves we directly see that the "spectroscopic" and "visual" characteristics have the same effect. On the other hand, the curve for the stars without further companions rises less rapidly than the others, showing that "multiple" systems tend to harbour lighter planets. This result is however not statistically significant. The KolmogorovSmirnov probability that curves a and $d$ come from the same underlying population is 0.35 . The curves even become indistinguishable if we restrict the sample to periods smaller than 1 year, so avoiding the bias favouring massive planets with long periods for which an additional radial-velocity drift is harder to

\footnotetext{
${ }^{9}$ In order to decrease the statistical noise, the distributions in Figs. 7 and 8 are shown for all detected exoplanets. It has however been verified that the results do not change qualitatively when considering only the stars in the volume-limited CORALIE planet-search sample (41/78 stars).
} 


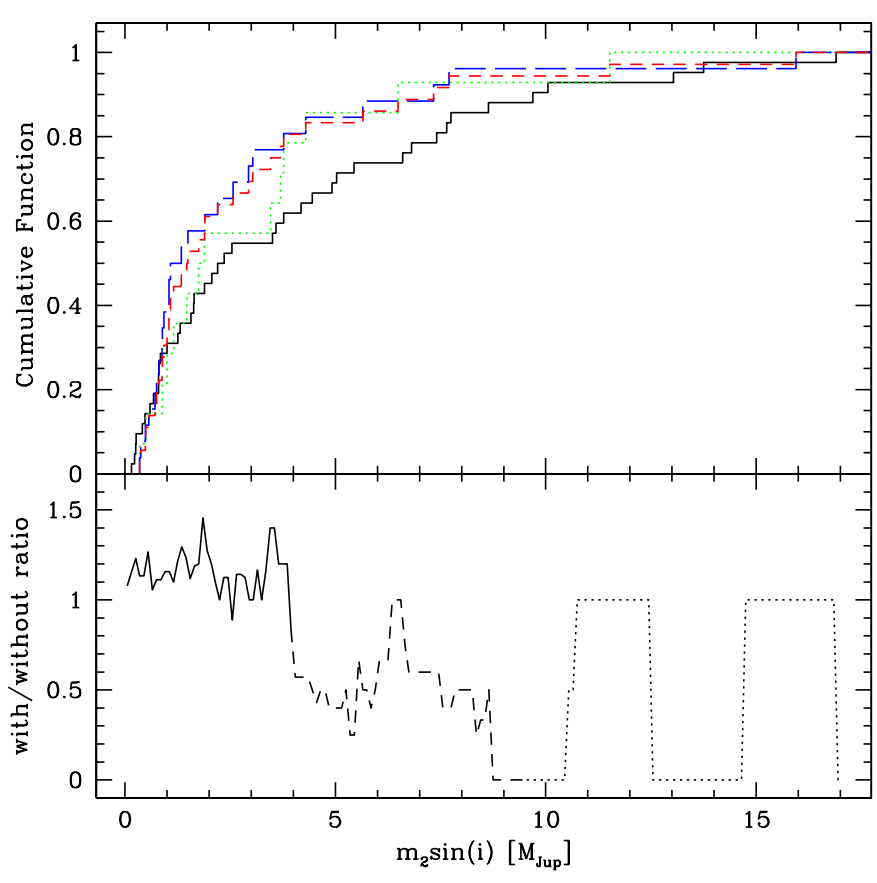

Fig. 7. Upper panel. Cumulative functions of the planetary mass distributions for stars hosting planets: a) without further visual or spectroscopic companion (solid line), b) with a visual companion (within 1 arcmin, dotted line), c) with an additional radial-velocity drift (longdash line) and d) with a visual or a spectroscopic companion (b) or c), short-dash line). Lower panel. Ratio of the number of stars hosting planets with visual or spectroscopic additional companions to the number of stars without further companion, in $2-M_{\text {Jup }}$ smoothing windows.

see than for short-period systems, over the typical span of the current planet-search programmes.

\subsection{Properties of mass subclasses}

It is worth noticing here that, in the lower panel of Fig. 7, the change in the mass regime is around $4 M_{\text {Jup }}$, at the same position as the limit between the two possible modes of the planetary mass distribution in Fig. 1 (lower panel). This suggests that the bimodality of the mass distribution is probably also not statistically significant and is potentially due to the mentioned observational bias. It is however interesting to check if the stars in the two mass subclasses show peculiar characteristics. This is achieved by comparing the distributions and cumulative functions of stellar and orbital properties for the two populations (limit at $4 M_{\text {Jup }}$, Fig. 8).

\subsubsection{Metallicity}

The metallicity distributions for the two mass subclasses are presented in the left column of Fig. 8. On average, the heavier companions seem to orbit stars slightly less metal rich than lighter planets (middle panel). The difference is however not statistically significant (KS prob $=0.061$, lower panel) as already pointed out by Santos et al. (2001a,b). The same result holds when restricting the sample to the CORALIE programme $(\mathrm{KS}$ prob $=0.065)$ and the same trend is also observed for the
Lick survey by Fischer et al. (2002) who compare the mean metallicities of two mass classes (limit at $5 M_{\text {Jup }}$ ). The difference originates mainly from a decrease with increasing metallicities of the distribution of stars with "massive" companions whereas the distribution for stars with lighter planets increases in the same metallicity range. An improvement of the available statistics will shed light on the question.

An interesting feature of the metallicity-mass distribution is the lack of massive planets at very low metallicities (upper left panel), although stars with massive planets are on average more metal poor than stars with light planets, as seen above. Below $[\mathrm{Fe} / \mathrm{H}] \simeq-0.25$, only HD $114762\left(m_{2} \sin i=11 M_{\text {Jup }}\right)$, often considered a brown dwarf (Cochran 1991), has a mass larger than $1.1 M_{\text {Jup }}(\otimes$ in the upper left panel of Fig. 8). On the low-metallicity side of the diagram, there even seems to be a limit imposing a minimum metallicity for the star to harbour a planet with a minimum given mass. This corroborates the idea that more "solid" material is needed in the accretion disk to form more massive planets. It also may be interpreted in terms of shorter time scales needed to accrete the planet core in metal rich environments, leaving more time for the planet growth over the lifetime of the disk. Both interpretations support the gas-accretion scenario for the formation of giant planets.

\subsubsection{Eccentricity-Period}

It has often been pointed out (e.g. Heacox 1999; Mayor \& Udry 2000; Udry et al. 2000b; Stepinski \& Black 2001, and references therein) that, for periods larger than a few tens of days, the eccentricity distributions of planetary systems and stellar binaries are unexpectedly similar. For shorter periods, evolutionary effects (planet migration, tidal circularization) change the distribution, favouring low- or zero-eccentricity orbits for both populations. The middle column of Fig. 8 shows that this is also true for the two defined subclasses of planetary masses. The distributions presented in the middle panel are very similar except for a prominent peak of "light" planets at small eccentricities. The latter corresponds to short-period, close-in planets, probably circularized through the migration process. They also relate to the accumulation at short periods in the middle panel of the 3 rd column in the figure. When restricting the sample to periods longer than 50 days, the mentioned peaks mainly responsible for the difference observed in the cumulative functions (KS-prob values in the figures) - disappear. Note however that the longer-period part of the distribution is still very much observationally biased.

An important point to note here is that massive planets on short-period orbits are rare - although they are more easily detected than lighter ones. If we consider the companion of HD 162020 ( $\otimes$ in the upper right panel) to be a brown dwarf as shown in Sect. 4, the only observed, short-period massive candidate is $\tau$ Boo, close to the chosen limit of the considered mass subclasses. While submitting this paper, we learned about a study by Zucker \& Mazeh (2002) estimating the statistical significance of this feature. 


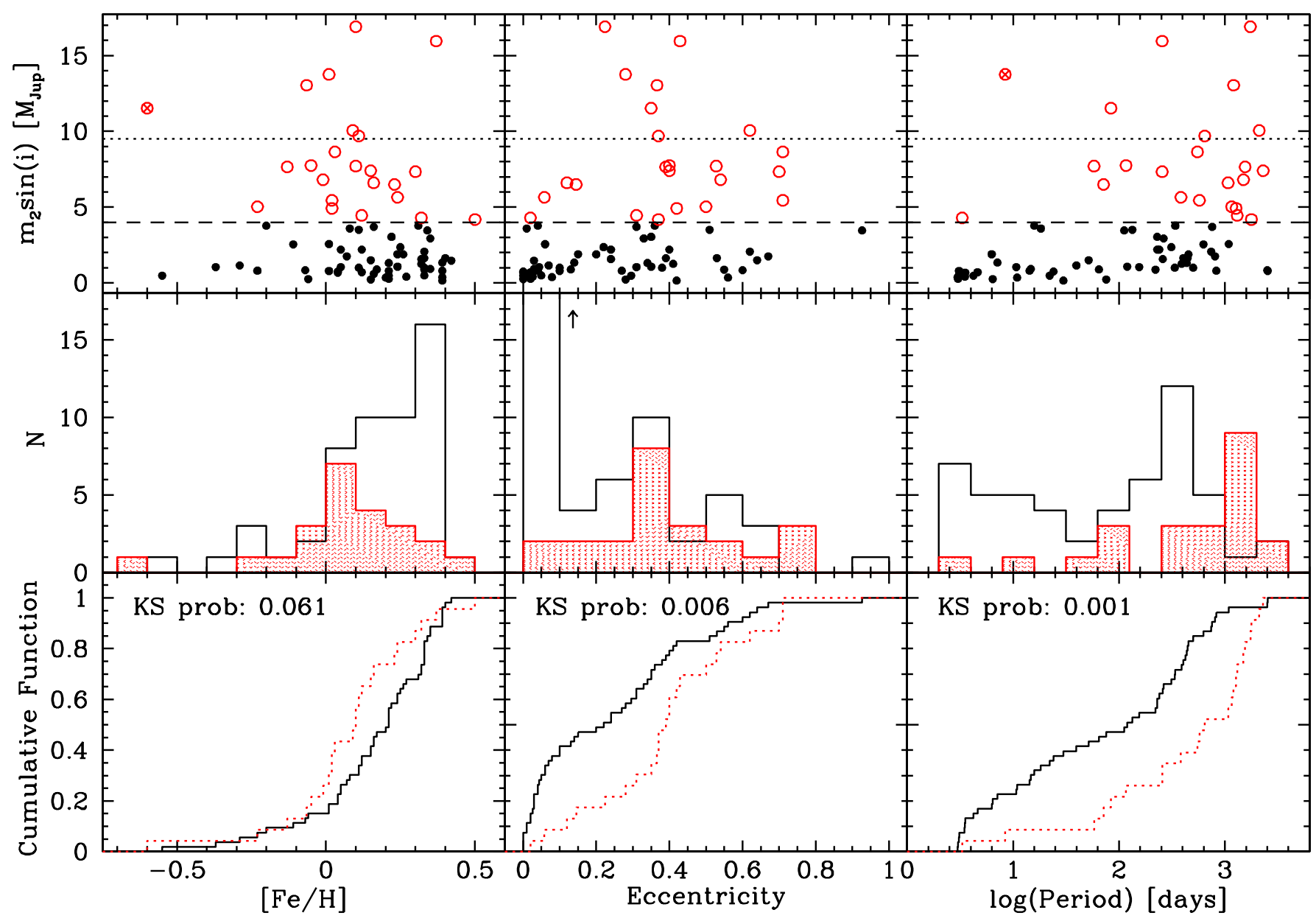

Fig. 8. Metallicity, eccentricity and period distributions of star hosting planets. Comparison between subsamples with different planetary masses: $m_{2} \sin i \leq 4 M_{\text {Jup }}$ (filled circles, open histogram and solid line) and $m_{2} \sin i>4 M_{\text {Jup }}$ (open circles, filled histogram and dotted line). The Kolmogorov-Smirnov probability that the two distributions come from the same underlying population is given in the lower panels. The probable brown dwarfs HD 114762 (in the upper-left panel) and HD 162020 (in the upper-right panel) are represented by crosses superimposed to open circles.

\subsubsection{Concluding remark}

We also have searched for differences between other distributions of orbital and stellar-host properties of "light" and "massive" exoplanets, without success. In particular, the primary masses do not correlate at all with the planet masses. The only observed trends are the need for metal-rich stars and the lack of short periods for massive planets. If confirmed with improved statistics, these features may bring constraints for the migration scenario. Possible explanations may invoke the idea that massive planets do not migrate as easily as lighter ones or, on the contrary, that they cannot stop their migration process when reaching the central part of the system, falling into the star. The higher metallicity of stars hosting light planets may support this latter view.

Acknowledgements. It is a pleasure to thank J.-P. Zahn for his useful comments on tidal dissipation theory and the referee for his remarks that helped to improve the paper. We are also grateful to the staff from the Geneva and Haute-Provence Observatories who have built and maintain the new 1.2-m Euler Swiss telescope and the CORALIE echelle spectrograph at La Silla. We thank the Geneva University and the Swiss NSF (FNRS) for their continuous support for this project.
Support to N.S. from Fundação para a Ciência e Tecnologia (Portugal) in the form of scholarships is gratefully acknowledged.

\section{References}

Baranne, A., Queloz, D., Mayor, M., et al. 1996, A\&AS, 119, 373 Boss, A. 2000, AAS/Division for Planetary Sciences Meeting Boss, A. 2001, ApJ, 563, 367

Bouchy, F., \& Carrier, F. 2001, A\&A, 374, L5

Bouchy, F., Pepe, F., \& Queloz, D. 2001, A\&A, 374, 733

Chelli, A. 2000, A\&A, 358, L59

Cochran, W. 1991, in NASA Reports of Planetary Astronomy, 153

Donahue, R. 1993, Ph.D. Thesis, New Mexico State University

Duquennoy, A., \& Mayor, M. 1991, A\&AS, 248, 485

ESA. 1997, The HIPPARCOS and TYCHO catalogue, ESA-SP 1200

ESO. 2000, Press Release: Exoplanet galore!,

http://www . eso.org/outreach/pressrel/pr2000/pr13Q0.html

Fischer, D., Marcy, G. W., Butler, R. P., et al. 2002, PASP, in press

Flower, P. 1996, ApJ, 469, 355

Ford, E., Rasio, F., \& Sills, A. 1999, ApJ, 514, 411

Goldreich, P., \& Keeley, D. A. 1977, ApJ, 211, 934

Gonzalez, G., Laws, C., Tyagi, S., \& Reddy, B. E. 2001, AJ, 121, 432 
Guillot, T., Burrows, A., Hubbard, W. B., Lunine, J. I., \& Saumon, D. 1996, ApJ, 459, L35

Halbwachs, J., Arenou, F., Mayor, M., \& Udry, S. 2000a, in IAU Symp. 200P, ed. B. Reipurth, \& H. Zinnecker, 132

Halbwachs, J.-L., Arenou, F., Mayor, M., Udry, S., \& Queloz, D. 2000b, A\&A, 355, 581

Heacox, W. 1999, ApJ, 526, 928

Henry, G. 1999, PASP, 111, 845

Henry, T., Soderblom, D., Donahue, R., \& Baliunas, S. 1996, AJ, 111, 439

Hugh, R., Butler, R., Tinney, C., et al. 2002, ApJ, submitted

Hut, P. 1980, A\&A, 92, 167

Jorissen, A., Mayor, M., \& Udry, S. 2001, A\&A, 379, 992

Kley, W. 2000, MNRAS, 313, L47

Latham, D., Stefanik, R., Mazeh, T., Mayor, M., \& Burki, G. 1989, Nature, 339, 38

Lloyd, J., Liu, M., Graham, J., et al. 2000, in Planetary Systems in the Universe: Observations, Formation and Evolution, ed. A. Penny, P. Artymowicz, A.-M. Lagrange, \& S. Russell, IAU Symp. 202, ASP Conf. Ser., in press

Lucas, P., \& Roche, P. 2000, MNRAS, 314, 858

Luhman, K., \& Jayawardhana, R. 2002, ApJ, 566, 1132

Marcy, G., Butler, R., Vogt, S., Fischer, D., \& Liu, M. 1999, ApJ, 520, 239

Marcy, G., Butler, R., Vogt, S., et al. 2001, ApJ, 555, 418

Marcy, G., Butler, R., Williams, E., et al. 1997, ApJ, 481, 926

Mayor, M., \& Queloz, D. 1995, Nature, 378, 355

Mayor, M., Queloz, D., Udry, S., \& Halbwachs, J.-L. 1997, Astronomical and biochemical origins and search for life in the Universe, ed. R. Cosmovici, S. Browyer, \& D. Werthimer, in IAU Coll., 161, 313

Mayor, M., \& Udry, S. 2000, Disks, Planetesimals and Planets, ed. F. Garzón, C. Eiroa, D. de Winter, \& T. Mahoney, in ASP Conf. Ser., 219, 441

Mayor, M., Udry, S., Halbwachs, J.-L., \& Arenou, F. 2001, in The Formation of Binary Stars, ed. H. Zinnecker, \& R. Mathieu, IAU Symp., 200, 45

Murray, N., Chaboyer, B., Arras, P., Hansen, B., \& Noyes, R. W. 2001, ApJ, 555, 801

Noyes, R., Hartmann, L., Baliunas, S., Duncan, D., \& Vaughan, A. 1984, ApJ, 279, 763

Pepe, F., Mayor, M., Galland, F., et al. 2002, A\&A, 388, 632
Queloz, D., Allain, S., Mermilliod, J.-C., Bouvier, J., \& Mayor, M. 1998, A\&A, 335, 183

Queloz, D., Eggenberger, A., Mayor, M., et al. 2000a, A\&A, 359, L13

Queloz, D., Henry, G. W., Sivan, J. P., et al. 2001a, A\&A, 379, 279

Queloz, D., Mayor, M., Naef, D., et al. 2000b, in From extrasolar planets to cosmology, ed. J. Bergeron, \& A. Renzini, ESO Astrophys. Symp., 548

Queloz, D., Mayor, M., Udry, S., et al. 2001b, The Messenger, 105, 1

Rasio, F., Tout, C., Lubow, S., \& Livio, M. 1996, ApJ, 470, 1187

Saar, S., Butler, R., \& Marcy, G. 1998, ApJ, 498, L153

Saar, S., \& Donahue, R. 1997, ApJ, 485, 319

Santos, N. C., Israelian, G., \& Mayor, M. 2001a, A\&A, 373, 1019

Santos, N. C., Israelian, G., \& Mayor, M. 2001b, in the 12th Cambridge workshop on Cool Stars, Stellar Systems, and the Sun, ASP Conf. Ser., in press

Santos, N. C., Mayor, M., Naef, D., et al. 2000, A\&A, 361, 265

Schaerer, D., Charbonnel, C., Meynet, G., Maeder, A., \& Schaller, G. 1993, A\&AS, 102, 339

Schaller, G., Schaerer, D., Meynet, G., \& Maeder, A. 1992, A\&AS, 96, 269

Stepinski, T., \& Black, D. 2001, A\&A, 371, 250

Udry, S., \& Mayor, M. 2001, in Astrobiology, The Quest for the Conditions of Life, ed. G. Horneck, \& C. Baumstark-Khan (Springer Verlag), 25

Udry, S., Mayor, M., Naef, D., et al. 2000a, A\&A, 356, 590

Udry, S., Mayor, M., \& Queloz, D. 2000b, in Planetary Systems in the Universe: Observations, Formation and Evolution, ed. A. Penny, P. Artymowicz, A.-M. Lagrange, \& S. Russell, IAU Symp. 202, ASP Conf. Ser., in press

Vaughan, A. H., Preston, G. W., \& Wilson, O. C. 1978, PASP, 90, 267

Verbunt, F., \& Phinney, E. 1995, A\&A, 296, 709

Zahn, J.-P. 1989, A\&A, 220, 112

Zahn, J.-P. 1992, in Binaries as Tracers of Stellar Formation, Bettmeralp, Switzerland, ed. A. Duquennoy, \& M. Mayor (Cambridge University Press), 253

Zahn, J.-P. 1994, A\&A, 288, 829

Zahn, J.-P., \& Bouchet, L. 1989, A\&A, 223, 112

Zapatero Osorio, M. R., Béjar, V. J. S., Martín, E. L., et al. 2000, Science, 290, 103

Zucker, S., \& Mazeh, T. 2001, ApJ, 562, 1038

Zucker, S., \& Mazeh, T. 2002, ApJL, in press [astro-ph/0202415] 